\title{
Pleckstrin homology domain of phospholipase D2 is a negative regulator of focal adhesion kinase
}

\author{
Mi Kyoung Kim ${ }^{1}$, Won Chan Hwang ${ }^{1,2}$ \& Do Sik Min ${ }^{2, *}$ \\ ${ }^{1}$ Department of Molecular Biology, College of Natural Science, Pusan National University, Busan 46241 , ${ }^{2}$ College of Pharmacy, Yonsei \\ University, Incheon 21983, Korea
}

\begin{abstract}
Phospholipase D2 (PLD2) has been implicated in the tyrosine kinase-mediated signaling pathways, but the regulation events are yet to be identified. Herein, we demonstrate that pleckstrin homology (PH) domain of PLD2 (PLD2-PH) exerts an antitumorigenic effect via the suppression of PLD2 and focal adhesion kinase (FAK). The kinase domain of FAK interacts with PLD2-PH and induces tyrosine phosphorylation and activation of PLD2. Furthermore, PLD2 increased tyrosine phosphorylation of FAK. However, ectopic expression of the PLD2-PH competes for binding to FAK and reduces the interaction between PLD2 and FAK, thereby suppressing FAK-induced PLD activation and tyrosine phosphorylation of FAK. The PLD2-PH suppressed the migration and invasion of glioblastoma cells, as well as tumor formation in a xenograft mouse model. This study uncovers a novel role of PLD2-PH as a negative regulator of PLD2 and FAK. [BMB Reports 2021; 54(2): 112-117]
\end{abstract}

\section{INTRODUCTION}

Phospholipase D (PLD) catalyzes the hydrolysis of phospholipids to generate phosphatidic acid (PA). Phosphatidylcholinespecific PLD isoforms, PLD1 and PLD2, contain phox homology (PX) and pleckstrin homology $(\mathrm{PH})$ domains, which are both involved in interactions with other proteins (1-3). By producing the signaling molecule PA, PLD plays a role in many diverse physiological processes, including proliferation, secretion, cytoskeletal reorganization, and vesicle trafficking (2). PLD is activated by various tyrosine kinases (4-9), mitogenic Wnt signaling $(5,7,10)$, and $G$ protein-coupled receptors (10). Dysregulation of PLD is associated with an increase in cell invasion, angiogenesis, evasion of growth suppressors, resistance to cell death,

*Corresponding author. Tel: +82-32-749-4522; Fax: +82-32-749-4105; E-mail:minds@yonsei.ac.kr

https://doi.org/10.5483/BMBRep.2021.54.2.154

Received 21 July 2020, Revised 10 August 2020, Accepted 19 August 2020

Keywords: Focal adhesion kinase, Phospholipase D2, Pleckstrin homology domain, Tumorigenesis, Tyrosine phosphorylation and metastasis (1-4). Furthermore, PLD exerts its biological functions via specific interactions with its binding partners, regardless of its lipase activity (11). The PX domain of PLD2 promotes the GTPase activity of dynamin by acting as a GTPase-activating protein (12) and also stimulates small G proteins by acting as a guanine nucleotide exchange factor (13). The $\mathrm{PH}$ domain of PLD1 (PLD1-PH) interacts with and accelerates the degradation of hypoxia-inducible factor 1 -alpha (HIF-1 $\alpha$ ), thus suppressing tumor progression (14). Tyrosine phosphorylation is commonly associated with the regulation of the mitogenic signaling pathways. Receptor and non-receptor tyrosine kinases induce the tyrosine phosphorylation of PLD2 and stimulate PLD activity $(2,9,15)$. However, it is unclear whether the tyrosine phosphorylation of PLD2 is required for the activation of PLD2.

Focal adhesion kinase (FAK) is responsible for the tyrosine phosphorylation of paxillin (16). The overexpression of wild-type (WT) PLD2, but not a catalytically inactive mutant of PLD2, reduced ATP-induced FAK activation and paxillin phosphorylation (17), suggesting that the enzymatic activity of PLD2 is involved in the negative regulation of the ATP-induced phosphorylation of FAK and paxillin. However, in this study, we found that the interaction between PLD2 and FAK induces the mutual activation of these enzymes via a positive feedback loop. Furthermore, the PH domain of PLD2 (PLD2-PH) can interact with FAK but disrupt the positive feedback loop between them by inhibiting their interaction, leading to suppressed migration and invasion of cancer cells, as well as reduced tumor formation.

\section{RESULTS}

FAK predominantly induces the tyrosine phosphorylation of PLD2 and interacts with PLD2

We first examined whether FAK affects the tyrosine phosphorylation of PLD isozymes. After cotransfection of FAK with PLD isozymes in HEK293 cells, the lysates were coimmunoprecipitated and immunoblotted with antibodies to P-Tyr and PLD. The antibody we used for detecting PLD recognizes both PLD1 and PLD2 (18). We observed that PLD2 was tyrosinephosphorylated more strongly by FAK than PLD1 (Fig. 1A). In fact, PLD1 was tyrosine-phosphorylated very weakly by FAK. 
A

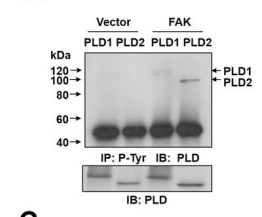

B

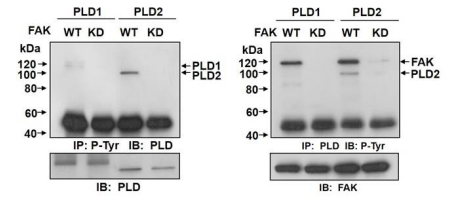

C
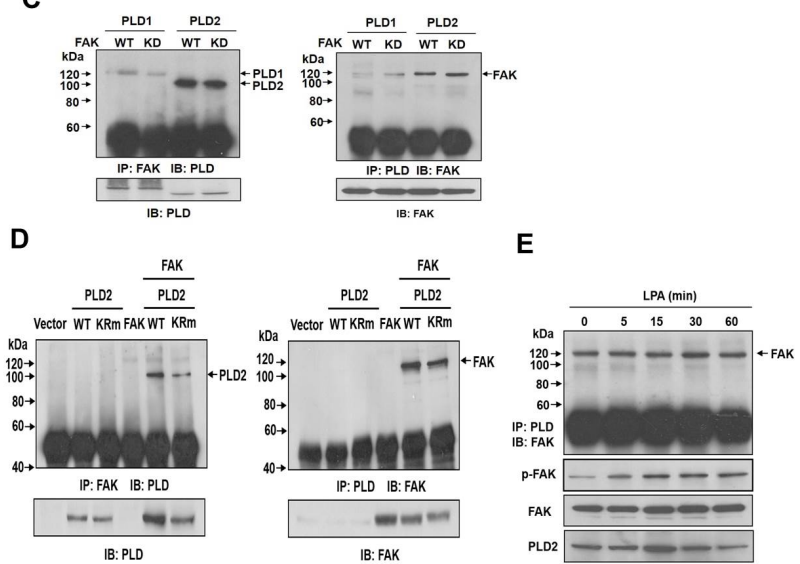

Fig. 1. FAK preferentially binds PLD2 and induces tyrosine phosphorylation. (A-D) HEK293 cells were co-transfected with the indicated constructs, and the cell lysates were immunoprecipitated and/or immunoblotted with the indicated antibodies. (E) HN33 cells were treated with LPA $(100 \mu \mathrm{M})$ for the indicated time, and the interaction of PLD2 with FAK was analyzed by immunoprecipitation and immunoblotting. The data are representative of three independent experiments.

Thus, it seems that PLD2 rather than PLD1 is preferentially tyrosine phosphorylated by FAK. The kinase-defective (KD) mutant of FAK (K454R) did not induce tyrosine phosphorylation of any of the PLD isozymes, as expected (Fig. 1B). Next, we wanted to further characterize how tyrosine phosphorylation of PLD isozymes occurs via their interaction with FAK. Although both PLD1 and PLD2 interacted with the FAK-WT and FAK-KD proteins, PLD2 demonstrated a stronger interaction with FAK compared to PLD1 (Fig. 1C). The catalytic activity of FAK did not affect the interaction with PLD, as both FAK-WT and FAK-KD were capable of interacting equally with the PLD isozymes (Fig. 1C). FAK was also observed to associate with both the WT and the catalytically inactive mutant of PLD2 (PLD2-KRm) that lacks lipase activity (Fig. 1D). Furthermore, lysophosphatidic acid (LPA), which activates FAK, did not affect the interaction between PLD2 and FAK in HN33 (hippocampal neuronal) cells predominantly expressing endogenous PLD2 (Fig. 1E), suggesting that the interaction of the proteins is ligand-independent. Overall, these results suggest that PLD2 is tyrosine-phosphorylated via an interaction with FAK.

\section{The PLD2-PH domain suppresses FAK-induced PLD activation by inhibiting the interaction between FAK and PLD2}

FAK contains three functional domains: the four-point-one, ezrin, radixin, moesin (FERM) domain, the kinase domain, and the focal adhesion targeting (FAT) domain (Fig. 2A, upper). To identify the domain of FAK responsible for the interaction with PLD2, HEK293 cells were co-transfected with PLD2 and HAtagged versions of the FAK FERM, kinase, or FAT domains. Immunoprecipitation and immunoblot analyses showed that the kinase domain of FAK interacted with PLD2 (Fig. 2A, lower). Next, we used glutathione-S-transferase (GST)-PLD2 fragment fusion proteins to determine the PLD2 region involved in the binding interaction with FAK (Fig. 2A, upper). The GST pull-down assays showed that FAK strongly binds to the regions of GST-PLD2 covering amino acids 1-314 (F1) and 315-475 (F2) (Fig. 2B). We further examined whether the F1 or F2 fragment of PLD2 controls the association of PLD2 with FAK. Interestingly, the ectopic expression of the F1 fragment of PLD2 (PLD2-F1) dramatically suppressed the interaction between PLD2 and FAK in a dose-dependent manner, whereas the F2 fragment of PLD2 (PLD2-F2) had only a marginal effect on their interaction (Fig. 2C). These results indicate that the F1 fragment, but not the F2 fragment, of PLD2 likely competes with FAK for the binding of WT PLD2. Therefore, we hypothesized that the F1 region of PLD2 containing the PX and PH domains are important for the interaction with FAK. We next wanted to determine whether FAK binds to the $\mathrm{PX}$ or $\mathrm{PH}$ domain of PLD2. We observed that PLD2 with a deleted PX domain in the $\mathrm{F} 1$ fragment ( $\mathrm{F} 1 \Delta \mathrm{PX})$ was still able to interact with FAK. This result indicated that FAK was not binding to the PX domain of PLD2 (Fig. 2D), but to the PH domain of PLD2. Further supporting this conclusion, the ectopic expression of the PLD2-PH domain suppressed the association of PLD2 with FAK (Fig. 2E). We also examined the effects of FAK-induced tyrosine phosphorylation of PLD2 on PLD activity. Both FAKWT and FAK-KD were observed to increase PLD activity in HN33 cells expressing endogenous PLD2 but not PLD1 (Fig. $2 \mathrm{~F})$, suggesting that the tyrosine phosphorylation of PLD2 by FAK is not involved in PLD2 activation. Furthermore, the kinase domain of FAK, but not the FERM or FAT domain, stimulated PLD activity (Fig. 2G), indicating that some of the interactions between PLD2 and FAK are involved in PLD activation. Interestingly, the ectopic expression of the PLD2-PH domain inhibited the FAK-induced activation of PLD2 in HN33 cells (Fig. $2 \mathrm{H}$ ). These results suggest that the PLD2-PH domain may inhibit FAK-induced PLD2 activation by disrupting the interaction between FAK and PLD2 via competition for binding to FAK.

The PLD2-PH domain suppresses the tyrosine phosphorylation of FAK that is normally induced by PLD2 The phosphorylation status of specific FAK residues is known to regulate specific cellular processes. For example, the autophosphorylation of FAK at Tyr397 plays a crucial role in the overall 


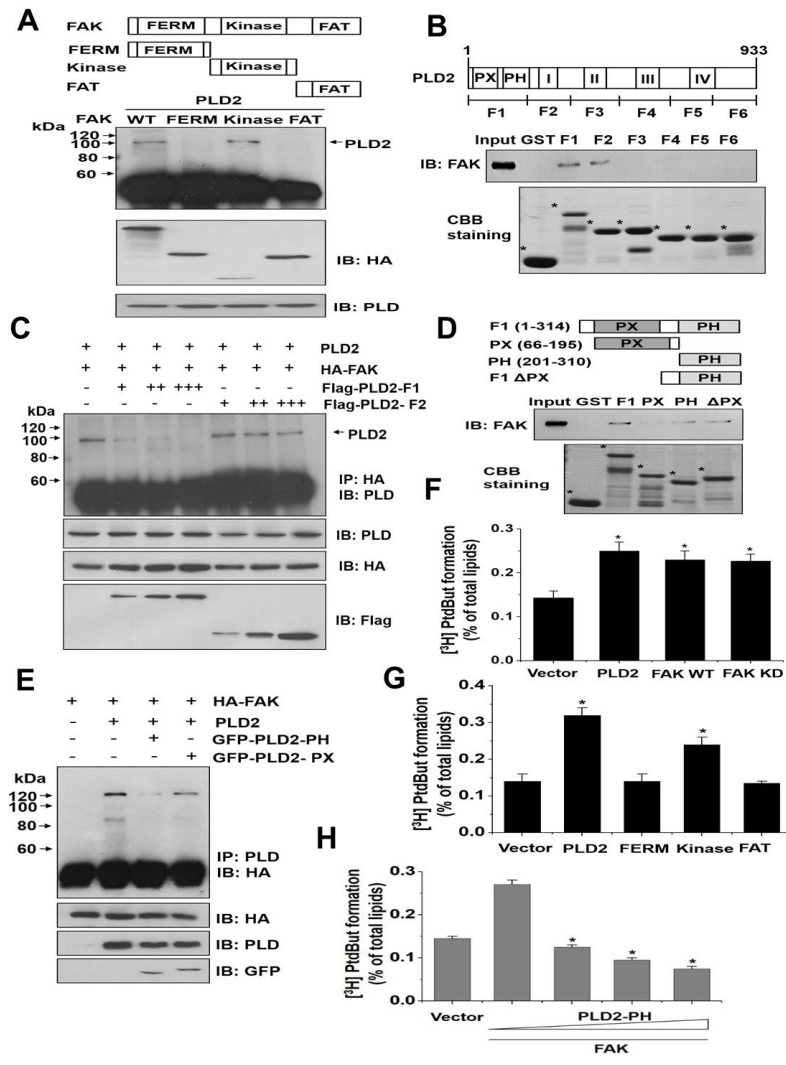

Fig. 2. The PLD2-PH domain suppresses FAK-induced PLD2 activation by reducing the interaction between FAK and PLD2. (A) Schematic representation of full-length WT FAK, as well as its FERN, kinase, and FAT domains. HEK293 cells were transfected with the indicated constructs, and the lysates were subjected to immunoprecipitation and/or immunoblotting with the indicated antibodies. (B) Schematic representation of the structure of PLD2, including its PX, PH, and I-IV domains (conserved domains in the PLD family). A GST pull-down assay using equal amounts of GST or GSTPLD2 fragment fusion proteins immobilized on Glutathione Sepharose 4B beads was performed on the lysates of HEK293 cells. The amount of GST fusion proteins was visualized by Coomassie Brilliant Blue (CBB) staining. (C) The effect of PLD2-F1 or PLD2F2 on the interaction between PLD2 and FAK. (D) The effect of the PX or PH domains of PLD2 on its binding to FAK. Domain mapping for the interaction site between PLD2 and FAK was performed using a GST-pull down assay. (E) The effect of the PLD2-PH domain on the interaction between PLD2 and FAK. (F-H) The effect of the transfection of the indicated constructs on PLD2 activity in HN33 cells. ${ }^{*} P<0.05$ compared to the cells transfected with the empty vector. The results are shown as the mean \pm SEM. Immunoblotting data are representative of three independent experiments.

activation of FAK by providing a docking site for Src tyrosine kinase to bind and phosphorylate FAK (19). In turn, the phosphorylation of FAK at Tyr576 and Tyr577 by Src increases the maximum catalytic activity of FAK (19). Src also phosphorylates FAK at Tyr861 (20). Paxillin is phosphorylated by activated
A

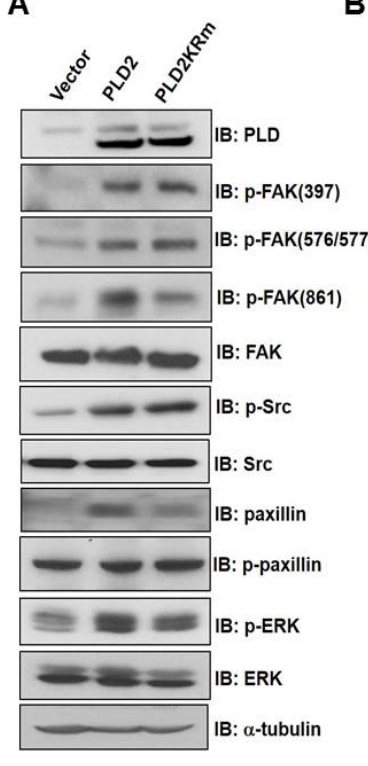

B

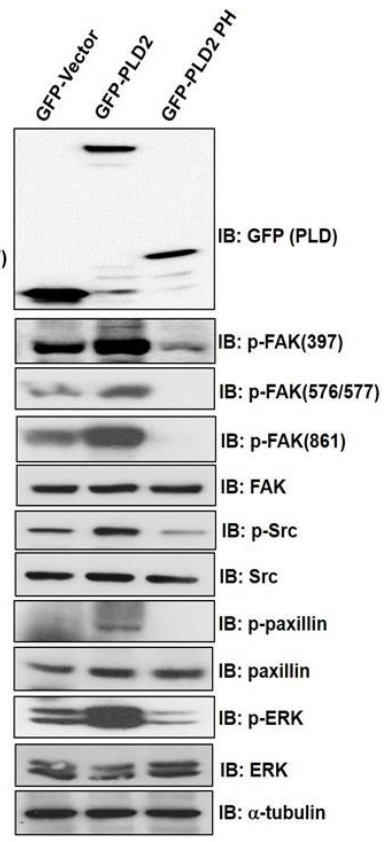

Fig. 3. The PLD2-PH domain suppresses the tyrosine phosphorylation of FAK that is normally induced by PLD2. (A) The lysates of U87MG cells expressing WT PLD2 and catalytically inactive PLD2 (PLD2-KRM) were analyzed by immunoblotting with the indicated antibodies. (B) The effect of the PLD2-PH domain on the activation of FAK, as analyzed through immunoblotting. The data are representative of three independent experiments.

FAK and is involved in the turnover of focal adhesions and cell motility (21). The activation of FAK also results in the activation of ERK. Therefore, we investigated whether PLD2 affects the phosphorylation of FAK and other FAK-mediated downstream signaling molecules. Interestingly, the expression of either WT PLD2 or PLD2-KRm increased the autophosphorylation of FAK Tyr397, the phosphorylation of FAK Tyr576/577 and FAK Tyr861 by Src, the phosphorylation of paxillin Tyr118, and the activation of ERK (Fig. 3A), suggesting that PLD activity is not required for the tyrosine phosphorylation and activation of FAK. Since the PLD2-PH domain inhibits the interaction of FAK with PLD2, we examined whether the PLD2-PH domain affects the activation of FAK and its downstream signaling molecules. We observed that the expression of the PLD2-PH domain reduced the phosphorylation of FAK (Tyr397, Tyr576/ 577, and Tyr861), the phosphorylation of Src (Tyr416) and paxillin (Tyr118), and the activation of ERK (Fig. 3B). Overall, these results suggest that PLD2 increases the tyrosine phosphorylation of FAK independent of its lipase activity and that the PLD2-PH domain suppresses the activation of FAK, probably by inhibiting the interaction between FAK and PLD2. 
The PLD2-PH domain suppresses the migration and invasion of cancer cells, as well as tumor growth

FAK mediates a number of signaling pathways associated with cell adhesion, mitogenesis, and oncogenic transformation $(22,23)$. Furthermore, overexpression of FAK can contribute to the development of several human malignancies. Therefore, we examined whether PLD2 and its PH domain can affect the migration and invasion of cancer cells. The ectopic expression of PLD2 or FAK increased the migration and invasion of U87MG glioblastoma cells, but the PLD2-PH domain significantly decreased migration and invasion of the glioblastoma cells (Fig. 4A and 4B). Since the PLD2-PH domain decreases the phosphorylation of FAK, as well as the migration and invasion of cancer cells, we examined whether the PLD2-PH domain could affect tumor growth. U87MG glioblastoma cells stably expressing the PLD2-PH domain were injected into mice to generate xenograft tumors. The injection of PLD2-PHexpressing U87MG cells resulted in decreased tumor growth compared to that of the vector-transfected U87MG cells (Fig. 4C). The PLD2-PH domain decreased the proliferation of the tumors according to immunohistochemistry results obtained using an antibody against Ki67, a biomarker for proliferation (Fig. 4D). Overall, these results suggest that the PLD2-PH domain suppresses the migration and invasion of cancer cells, as well as tumor formation.

\section{DISCUSSION}

In this study, we found that PLD2 and FAK positively regulates with each other and that the PLD2-PH domain is a negative regulator of this feedback loop. This study aimed to better define the molecular link between PLD2 and FAK and the role of this interaction in cancer. FAK induces tyrosine phosphorylation of PLD2 via the interaction of its kinase domain with PLD2. This interaction allows FAK to increase PLD2 activity independent of its kinase activity. Furthermore, PLD2 also stimulates the kinase activity of FAK. Until now, the molecular link between FAK and PLD had been poorly defined. Although PLD2 had been reported to be tyrosine-phosphorylated by a range of tyrosine kinases (such as epidermal growth factor receptor (EGFR), platelet-derived growth factor receptor (PDGF), Src, and JAK3 (2), it was unclear whether tyrosine phosphorylation of PLD2 was required for PLD activity. These findings indicate that FAK-induced tyrosine phosphorylation of PLD2 is not associated with PLD activation, because the catalytically inactive mutant of FAK was still able to stimulate PLD activity. The future identification and mutation of the tyrosine residues of PLD2 that are phosphorylated by FAK will be helpful in understanding the mechanism in which PLD2 is regulated by tyrosine phosphorylation. Interestingly, the PLD2-PH domain inhibited the activation of FAK and PLD2, probably by competitively suppressing the interaction between FAK and PLD2. Alternatively, it is still unclear how PLD2 induces the tyrosine phosphorylation of FAK. One possibility is that PLD2 can recruit
A

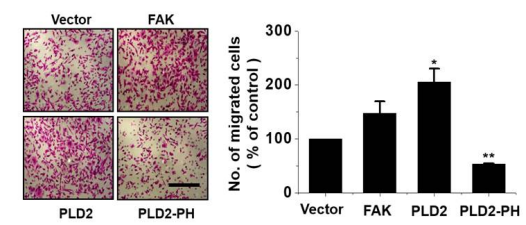

B

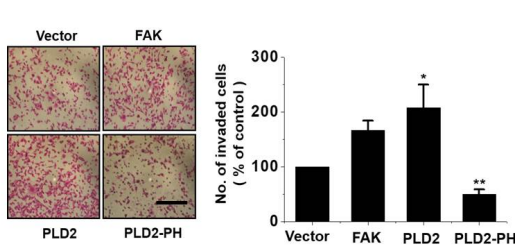

C

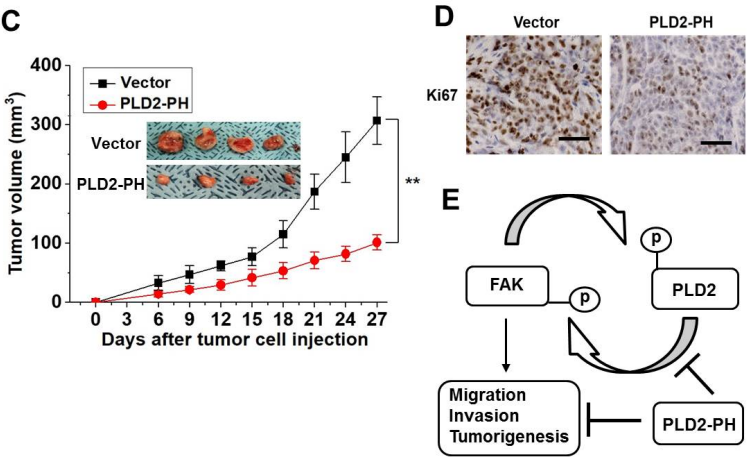

Fig. 4. The PLD2-PH domain suppresses the migration and invasion of cancer cells, as well as tumor growth. (A) Cell migration assays were conducted using Transwell plates for $24 \mathrm{~h}$ after transfection of the indicated constructs into U87MG cells. (B) Invasion assays were performed for $24 \mathrm{~h}$ using Matrigel-coated Transwell plates. The extent of cell migration and invasion was expressed as the average number of cells per field of view among the different experimental groups. Bar, $100 \mu \mathrm{m}$. ${ }^{*} \mathrm{P}<0.05$ compared to the empty vector. (C) U87MG cells expressing the empty vector or the PLD2-PH domain were injected into nude mice, and the tumor volume was then analyzed. Data are expressed as the mean \pm SD of eight different mice per group. (D) Immunohistochemical staining of Ki67 in the tumor tissues. Bar, $100 \mu \mathrm{m}$ Data are representative of three independent experiments. (E) Schematic representation for role of PLD2-PH. FAK and PLD2 are positively regulated with each other via tyrosine phosphorylation. PLD2-PH inhibits their interaction and activities, followed by suppression of migration, invasion and tumor formation.

specific protein kinases to FAK that induce the tyrosine phosphorylation of FAK. Further studies will be needed to fully investigate the mechanism of FAK-mediated PLD activation. Recently, it was reported that the PLD1-PH domain interacts with and promotes the degradation of HIF- $1 \alpha$ even under hypoxic conditions, thus suppressing tumor progression (14). In addition, the PLD1-PH domain itself increases the endocytosis of EGFR via the upregulation of rabaptin-5 (24). The interaction of PLD1 with HIF-1 $\alpha$ is involved in EGFR endocytosis (25). PLD2-PX domain acts as a GTPase-activating protein of dynamin (12). The PH and PX domains of the PLD isozymes may perform various functions depending on their 
different binding partners. PLD2-PH domain plays an antitumorigenic role by negatively regulating the interaction between PLD2 and FAK that normally results in their mutual activation through a positive feedback loop, acting as a potential target for the future development of novel cancer therapeutics (Fig. $4 \mathrm{E})$. It is speculated that under situation of high expression levels of PLD2 and FAK such as cancers, PLD2-PH domain might play a dominant role in the disruption of their interaction and activities. In conclusion, this study advances the understanding of the crosstalk that occurs between PLD2 and FAK during their activation via a positive feedback loop.

\section{MATERIALS AND METHODS}

\section{Cell lines and reagents}

The HEK293 (immortalized human embryonic kidney cells), HN33 (immortalized rat hippocampal neuronal cells), and U87MG (immortalized human glioblastoma cells) cell lines were cultured at $37^{\circ} \mathrm{C}$ in Dulbecco's modified Eagle's medium (DMEM, Gibco-BRL, CA, USA) containing 10\% fetal bovine serum (FBS) and 1\% antibiotic-antimycotic solution. The U87MG cells stably overexpressing the WT or the catalytically inactive mutant (K758R) of PLD2 (PLD2-KRm) were used as reported previously (26). PLD2-PH-expressing U87MG cells were established by transfection using Lipofectamine and selected using G418 $(700 \mu \mathrm{g} / \mathrm{ml})$. Protein A-Sepharose and Glutathione Sepharose $4 \mathrm{~B}$ were obtained from Amersham Biosciences (UK). The phosphatidylbutanol (PtdBut) standard was acquired from Avanti Polar Lipids, Inc. (AL, USA). [9, 10- $\left.{ }^{3} \mathrm{H}\right]$ myristic acid was purchased from Perkin-Elmer Life Sciences (MA, USA). The silica gel 60A thin-layer chromatography plates were obtained from Whatman (UK). The ECL Western Blotting Detection Kit was procured from Amersham Biosciences (UK).

\section{Immunoprecipitation and Immunoblotting}

The cell lysates were analyzed by immunoprecipitation and immunoblotting, as previously described (26). Enhanced chemiluminescence was used for signal detection. The following antibodies were used for immunoblotting: anti-phosphotyrosine (Upstate Biotechnology, NY, USA); anti-FAK, anti- $\alpha$-tubulin, anti-GFP, and anti-HA (Santa Cruz Biotechnology, TX, USA); anti-phospho-FAK (pY397, pY861, pY576/577), anti-phosphoTyr416-c-Src, anti-phospho-ERK, and anti-phospho-paxillin (Cell Signaling Technology, MA, USA); and anti-Ki67 (Abcam, UK). A polyclonal antibody that recognizes both PLD1 and PLD2 was generated, as previously described (18).

\section{GST pull-down assay}

Escherichia coli BL21 cells were transformed with the individual expression vectors encoding the glutathione-S-transferase (GST) fusion proteins. The generated GST fusion proteins were then purified using Glutathione Sepharose 4B medium. The lysates were incubated with the GST fusion proteins immobilized on Glutathione Sepharose 4B beads for $1 \mathrm{~h}$ at $4^{\circ} \mathrm{C}$. The bound proteins were then analyzed via immunoblotting.

\section{PLD activity assay}

PLD activity was determined by measuring the formation of $\left[{ }^{3} \mathrm{H}\right] \mathrm{PtdBut}$, the product of PLD-mediated transphosphatidylation, in the presence of 1-butanol. The cells were serum-starved in the presence of $3 \mu \mathrm{Ci}$ of $\left[9,10^{3} \mathrm{H}\right]$ myristic acid/ml for $12 \mathrm{~h}$. The cells were then washed, and $0.3 \%$ of 1 -butanol was added. The extraction and characterization of the lipids by thinlayer chromatography were performed as previously described (25).

\section{Migration and invasion assay}

Transwell plates with $8.0 \mu \mathrm{m}$ pores (Corning, NY, USA) were used for the migration assays. The cells were transfected transiently with the indicated constructs. Transfected cells suspended in $0.1 \mathrm{ml}$ serum-free DMEM were added to the top of each chamber. The bottom compartments of the Transwell chambers were filled with the migration-inducing medium (DMEM with $10 \%$ FBS) and then incubated at $37^{\circ} \mathrm{C}$ for $24 \mathrm{~h}$. The cells that migrated to the lower surface of the membranes were stained with hematoxylin and eosin and counted using a microscope. For the in vitro invasion assays, the upper surface of the Transwell membrane was coated with Matrigel $(10 \mu \mathrm{g} / \mathrm{ml})$, while the lower compartment of the chamber was filled with DMEM containing $10 \%$ FBS. The cells were placed in the upper compartment of each Transwell and incubated at $37^{\circ} \mathrm{C}$ for $24 \mathrm{~h}$. The cells invading to the lower compartment were stained with hematoxylin and eosin and counted using a microscope.

\section{Tumor formation and immunohistochemistry}

U87MG cells overexpressing PLD2-PH were injected into the right flank of nude mice. The diameters of the tumors in eight mice were measured at right angles ( $a=$ short and $b=$ long) using electronic calipers, and the tumor volumes were calculated using the formula volume $=\left(a \times b^{2}\right) / 5$. The Institutional Animal Care Committee of Pusan National University approved the animal studies (2013-0403). Immunohistochemical analyses were performed using an UltraTek horseradish peroxidase detection kit (ScyTek Laboratories, UT, USA). The diaminobenzidine-stained specimens were visualized using a general optical microscope with a camera (AxioCam ICc5; Carl Zeiss, Germany).

\section{Statistics}

The results are expressed as the mean \pm SD for the number of determinations indicated. Significant differences among the means were identified by ANOVA. A P-value $<0.05$ was considered significant.

\section{ACKNOWLEDGEMENTS}

This work was supported by a National Research Foundation 
of Korea (NRF) grant funded by the Korean government (NRF-2018R1A2B3002179, 2019M3A9A8065095) and by the Yonsei University Research Fund (2019-22-0193).

\section{CONFLICTS OF INTEREST}

The authors have no conflicting interests.

\section{REFERENCES}

1. Gomez-Cambronero J (2014) Phosphatidic acid, phospholipase D and tumorigenesis. Adv Biol Reg 54, 197-206

2. Bruntz RC, Lindsley CW and Brown HA (2014) Phospholipase D signaling pathways and phosphatidic acid as therapeutic targets in cancer. Pharmacol Rev 66, 10331079

3. Nelson RK and Frohman MA (2015) Physiological and pathophysiological roles for phospholipase D. J Lipid Res 56, 2229-2237

4. Min DS, Ahn BH and Jo YH (2001) Differential tyrosine phosphorylation of phospholipase D isozymes by hydrogen peroxide and the epidermal growth factor in A431 epidermoid carcinoma cells. Mol Cells 11, 369-378

5. Kang DW and Choi KY (2014) Functional regulation of phospholipase D expression in cancer and inflammation. J Biol Chem 289, 22575-22582

6. Zhao C, Du G, Skowronek K et al (2007) Phospholipase D2-generated phosphatidic acid couples EGFR stimulation to Ras activation by Sos. Nat Cell Biol 9, 706-712

7. Frohman MA (2015) The phospholipase D superfamily as therapeutic targets. Trends Pharmacol Sci 36, 137-144

8. Slaaby R, Jensen T, Hansen HS et al (1998) PLD2 complexes with the EGF receptor and undergoes tyrosine phosphorylation at a single site upon agonist stimulation. J Biol Chem 273, 33722-33727

9. Ahn BH, Kim SY, Kim EH et al (2003) Transmodulation between phospholipase D and c-Src enhances cell proliferation. Mol Cell Biol 23, 3103-3115

10. Brown HA, Thomas PG and Lindsley CW (2017) Targeting phospholipase $\mathrm{D}$ in cancer, infection and neurodegenerative disorders. Nat Rev Drug Disv 16, 351-367

11. Jang JH, Lee CS, Hwang D et al (2012) Understanding of the roles of phospholipase $\mathrm{D}$ and phosphatidic acid through their binding partners. Prog Lipid Res 51, 71-81

12. Lee CS, Kim IS, Park JB et al (2006) The phox homology domain of phospholipase D activates dynamin GTPase activity and accelerates EGFR endocytosis. Nat Cell Biol
$8,477-484$

13. Gomez-Cambronero J (2012) Biochemical and cellular implications of a dual lipase-GEF function of phospholipase D2 (PLD2). J Leuko Biol 92, 461-467

14. Park MH, Choi KY, Jung Y et al (2014) Phospholipase D1 protein coordinates dynamic assembly of HIF-1 $\alpha-\mathrm{PHD}-\mathrm{VHL}$ to regulate HIF-1 $\alpha$ stability. Oncotarget 5, 11857-11872

15. Henkels KM, Peng HJ, Frondorf K et al (2010) A comprehensive model that explains the regulation of phospholipase D2 activity by phosphorylation-dephosphorylation. Mol Cell Biol 30, 2251-2263

16. Schaller MD and Parsons JT (1995) pp125FAK-dependent tyrosine phosphorylation of paxillin creates a high-affinity binding site for Crk. Mol Cell Biol 15, 2635-2645

17. Bae YS and Ryu SH (2001) ATP-induced focal adhesion kinase activity is negatively modulated by phospholipase D2 in PC12 cells. Exp Mol Med 33, 150-155

18. Min DS, Ahn BH, Rhie DJ et al (2001) Expression and regulation of phospholipase $\mathrm{D}$ during neuronal differentiation of PC12 cells. Neuropharmacology 41, 384-391

19. Schaller MD, Hildebrand JD, Shannon JD et al (1994) Autophosphorylation of the focal adhesion kinase, pp125FAK, directs SH2-dependent binding of pp60src. Mol Cell Biol 14, 1680-1688

20. Mitra SK, Hanson DA and Schlaepfer DD (2005) Focal adhesion kinase: in command and control of cell motility. Nat Rev Mol Cell Biol 6, 56-58

21. Lim Y, Han I, Jeon J et al (2004) Phosphorylation of focal adhesion kinase at tyrosine 861 is crucial for Ras transformation of fibroblasts. J Biol Chem 279, 29060-29065

22. Schwock J, Dhani N and Hedley DW (2010) Targeting focal adhesion kinase signaling in tumor growth and metastasis. Expert Opin Ther Targets 14, 77-94

23. Jang HJ, Bak Y, Pham TH et al (2018) STK899704 inhibits stemness of cancer stem cells and migration via the FAKMEK-ERK pathway in HT29 cells. BMB Rep 51, 596-601

24. Park MH, Choi KY and Min DS (2015) The pleckstrin homology domain of phospholipase D1 accelerates EGFR endocytosis by increasing the expression of the Rab5 effector, rabaptin-5. Exp Mol Med 47, e200

25. Bligh EG and Dyer WJ (1959) A rapid method of total lipid extraction and purification. Can J Bioochem Physiol 37, 911-917

26. Park MH, Ahn BH, Hong YK et al (2009) Overexpression of phospholipase $\mathrm{D}$ enhances matrix metalloproteinase-2 expression and glioma cell invasion via protein kinase $\mathrm{C}$ and protein kinase $\mathrm{A} / \mathrm{NF}-\kappa \mathrm{B} / \mathrm{Sp} 1$-mediated signaling pathways. Carcinogenesis 30, 356-365 